\title{
A NONUNIFORM VERSION OF THE THEOREM OF RADON-NIKODYM IN THE FINITELY-ADDITIVE CASE WITH APPLICATIONS TO EXTENSIONS OF FINITELY-ADDITIVE SET FUNCTIONS
}

\author{
D. PLACHKY
}

(Communicated by Lawrence F. Gray)

\begin{abstract}
For $\mu, \nu \in b a_{+}(\Omega, \mathfrak{A})$ it is shown that the existence of a net of nonnegative functions $f_{\alpha^{\prime}}$ that are primitive relative to $\mathfrak{A}$ and satisfy $\lim _{\alpha} \int_{A} f_{\alpha} d \mu=\nu(A), A \in \mathfrak{A}$, is equivalent to the condition $\nu \lesssim \mu$, i.e. $\mu(A) \stackrel{=}{=} 0$ for some $A \in \mathfrak{A}$ implies $\nu(A)=0$. Furthermore, as an application it is proved that for $\mu, \nu \in b a_{+}(\Omega, \mathfrak{A})$ satisfying $\nu \lesssim \mu$ and any extension $\mu^{\prime} \in b a_{+}\left(\Omega, \mathfrak{A}^{\prime}\right)$ of $\mu$, where $\mathfrak{A}^{\prime}$ denotes some algebra of subsets of $\Omega$ containing $\mathfrak{A}$, there exists some extension $\nu^{\prime} \in b a_{+}\left(\Omega, \mathfrak{A}^{\prime}\right)$ of $\nu$ such that $\nu^{\prime} \lesssim \mu^{\prime}$ is valid.
\end{abstract}

Let $b a_{+}(\Omega, \mathfrak{A})$ denote the subset of the set $b a(\Omega, \mathfrak{A})$ of all bounded and finitely-additive set functions on the algebra $\mathfrak{A}$ of subsets of the set $\Omega$ that are nonnegative. Furthermore, $B(\Omega, \mathfrak{A})$ stands for the family of all real-valued functions on $\Omega$ that are uniform limits of functions of the type $\sum_{i=1}^{n} \alpha_{i} I_{A_{i}}$, $\alpha_{i} \in \mathbb{R}, A_{1} \in \mathfrak{A}, i=1, \ldots, n, n \in \mathbb{N}$ ("primitive function relative to $\mathfrak{A}$ "). Finally, $\nu$ is called weakly absolutely continuous with respect to $\mu$ (symbol: $\nu \lesssim \mu)$, if $\mu(A)=0$ for some $A \in \mathfrak{A}$ implies $\nu(A)=0$, and $\nu$ is called absolutely continuous with respect to $\mu$ (symbol: $\nu \ll \mu$ ), if for any $\varepsilon>0$ there exists some $\delta>0$ such $\nu(A)<\varepsilon$ whenever $\mu(A)<\delta$ for $A \in \mathfrak{A}$, where $\mu, \nu$ belong to $b a_{+}(\Omega, \mathfrak{A})$ (cf. [1, p. 159]). In the sequel the following equivalent condition for the relation $\nu \lesssim \mu, \mu, \nu \in b a_{+}(\Omega, \mathfrak{A})$, is used: $\int_{A} f d \mu \geq 0$ for all $A \in \mathfrak{A}$ for some $f \in B(\Omega, \mathfrak{A})$ implies $\int_{A} f d \nu \geq 0$ for all $A \in \mathfrak{A}$. This last condition is equivalent to the relation $\nu \lesssim \mu$, since one can replace $f \in B(\Omega, \mathfrak{A})$ by some function $f: \Omega \rightarrow \mathbb{R}$, which is primitive relative to $\mathfrak{A}$.

According to a well-known uniform version of the Radon-Nikodym theorem in the finitely-additive case due to Bochner, there exists a net $f_{\alpha}$ of nonnegative

Received by the editors February 8, 1990 and, in revised forms, May 20, 1990 and August 15, 1990.

1980 Mathematics Subject Classification (1985 Revision). Primary 28A12, 28A20.

Key words and phrases. Nonuniform version of the Radon-Nikodym theorem, finitely-additive set functions. 
functions that are primitive relative to $\mathfrak{A}$ and satisfy $\lim _{\alpha} \int_{A} f_{\alpha} d \mu=\nu(A)$ uniformly for $A \in \mathfrak{A}$ if and only if $\nu \ll \mu$ is valid (cf. [1, Theorem 6.3.4, p. 175] or [2, IV.9.14, p. 315]). Clearly, one might replace the net $f_{\alpha}$ by a sequence, whereas the notion of a net is essential for the following nonuniform version of the Radon-Nikodym theorem in the finitely-additive case:

Theorem. For $\mu, \nu \in b a(\Omega, \mathfrak{A})$ there exists a net of nonnegative functions $f_{\alpha}$ that are primitive relative to $\mathfrak{A}$ and satisfy $\lim _{\alpha} \int_{A} f_{\alpha} d \mu=\nu(A)$ for $A \in \mathfrak{A}$, if and only if $\nu \lesssim \mu$ is valid.

Proof. Clearly for a net $f_{\alpha}$ of nonnegative functions that are primitive relative to $\mathfrak{A}, \lim _{\alpha} \int_{A} f_{\alpha} d \mu=\nu(A), A \in \mathfrak{A}$, implies $\nu \lesssim \mu$. For the converse direction let $C$ denote the convex subset of $b a_{+}(\Omega, \mathfrak{A})$ consisting of all $\mu_{f} \in b a_{+}(\Omega, \mathfrak{A})$ defined by $\mu_{f}(A)=\int_{A} f d \mu, A \in \mathfrak{A}$, where $f$ belongs to the system $\mathfrak{F}$ of all nonnegative functions that are primitive with respect to $\mathfrak{A}$. Now the assumption that $\nu$ does not belong to the closure of $C$ with respect to the $B(\Omega, \mathfrak{A})$ topology of $b a(\Omega, \mathfrak{A})$ (cf. [1, Theorem 4.7.4] or [2, IV.5.1, p. 258]), implies the existence of some $g \in B(\Omega, \mathfrak{A})$ with the property $\int f g d \mu \leq c<c+\varepsilon \leq \int g d \nu$ for all $f \in \mathfrak{F}$ and some $c \in \mathbb{R}$ and $\varepsilon>0$, if one applies a well-known separation theorem for convex subsets (cf. [2, V.2.10, p. 417]) together with [2, V.3.12, p. 422]. In particular, $\int f g d \mu \leq 0$ for $f \in \mathfrak{F}$ is valid, since $\int f g d \mu>0$ for some $f \in \mathfrak{F}$ implies $\sup \left\{\int f g d \mu: f \in \mathfrak{F}\right\}=\infty$. Furthermore, $\int_{A} g d \mu \leq 0$, $A \in \mathfrak{A}$, implies $\int_{A} g d \nu \leq 0, A \in \mathfrak{A}$, because of $\nu \lesssim \mu$. This is a contradiction to $\int g d \nu \geq c+\varepsilon \geq \varepsilon$, since $c \geq 0$ is implied by $\int f g d \mu \leq c<c+\varepsilon \leq \int g d \nu$, $f \in \mathfrak{F}$, if one chooses $f=0$.

Remark. Under the additional assumption that $\mathfrak{A}$ is a $\sigma$-algebra of subsets of $\Omega$ (or more generally an algebra of subsets of $\Omega$ with the Seever property, cf. [1, p. 210]), the net of nonnegative functions $f_{\alpha}$ of the theorem can be replaced by a sequence if and only if the classical uniform version of the theorem of Radon-Nikodym (cf. [1, Theorem 6.3 .4$, p. 175] or [2, IV.9.14, p. 315]) is valid, i.e. $\nu \ll \mu$ holds true (cf. [1, Theorem 8.7.5, p. 225] and [2, V.3.14, p. 422]). The conclusion $\nu \ll \mu$ is no longer true if $\mathfrak{A}$ is only an algebra, as the special case shows, where $\mathfrak{A}$ is countable. In this situation $B(\Omega, \mathfrak{A})$ is separable, which implies that the underlying net of functions of the theorem may be replaced by a sequence (cf. [2, V.5.1, p. 426]).

A simple application of the theorem concerns the existence of extensions of $\mu, \nu \in b a_{+}(\Omega, \mathfrak{A})$ according to the following:

Corollary. For $\mu, \nu \in b a_{+}(\Omega, \mathfrak{A})$ satisfying $\nu \lesssim \mu$ and any extension $\mu^{\prime} \in$ $b a_{+}\left(\Omega, \mathfrak{A}^{\prime}\right)$ of $\mu$, where $\mathfrak{A}^{\prime}$ is some algebra of subsets of $\Omega$ containing $\mathfrak{A}$, there exists an extension $\nu^{\prime} \in b a_{+}\left(\Omega, \mathfrak{A}^{\prime}\right)$ of $\nu$ such that $\nu^{\prime} \lesssim \mu^{\prime}$ is valid.

Proof. Let $f_{\alpha}$ denote a net of nonnegative functions that are primitive relative to $\mathfrak{A}$ and satisfy $\lim _{\alpha} \int_{A} f_{\alpha} d \mu=\nu(A), A \in \mathfrak{A}$. Now the compactness of the unit ball of $b a\left(\Omega, \mathfrak{A}^{\prime}\right)$ with respect to the $B\left(\Omega, \mathfrak{A}^{\prime}\right)$-topology of $b a\left(\Omega, \mathfrak{A}^{\prime}\right)$ implies the existence of some subnet $f_{\beta}$ of the net $f_{\alpha}$ such that $\lim _{\beta} \int_{A^{\prime}} f_{\beta} d \mu^{\prime}$ 
exists for all $A^{\prime} \in \mathfrak{A}^{\prime}$ (cf. [2, V.4.2, p. 424] together with [2, IV.5.1, p. 258]), which defines some extension $\nu^{\prime} \in b a_{+}\left(\Omega, \mathfrak{A}^{\prime}\right)$ of $\nu$ satisfying $\nu^{\prime} \lesssim \mu^{\prime}$.

It might be interesting to find conditions that imply that the $f_{\alpha}$ of the underlying net of nonnegative functions of the theorem can be chosen to be already primitive relative to some given subalgebra $\overline{\mathfrak{A}}$ of $\mathfrak{A}$. The first example provides a sufficient condition of this type in terms of extensions of the restriction $\nu \mid \overline{\mathfrak{A}}$ of $\nu \in b a_{+}(\Omega, \mathfrak{A})$ to $\overline{\mathfrak{A}}$.

Example 1. Let $\mu, \nu \in b a_{+}(\Omega, \mathfrak{A})$ satisfy $\nu \lesssim \mu$. Then there exists a net $f_{\alpha}$ of nonnegative functions that are primitive relative to $\overline{\mathfrak{A}}$, where $\overline{\mathfrak{A}}$ denotes some subalgebra of $\mathfrak{A}$, such that $\lim _{\alpha} \int_{A} f_{\alpha} d \mu=\nu(A), A \in \mathfrak{A}$, is valid, if $\nu$ is the uniquely determined extension of $\nu \mid \overline{\mathfrak{A}}$ to $\mathfrak{A}$ among all $\lambda \in b a_{+}(\Omega, \mathfrak{A})$ satisfying $\lambda \lesssim \mu$ and $\lambda|\overline{\mathfrak{A}}=\nu| \overline{\mathfrak{A}}$. This follows similar to the proof of the corollary by applying [2, V.4.2, p. 424, IV.5.1, p. 258] to some net $f_{\alpha}$ of nonnegative functions that are primitive relative to $\overline{\mathfrak{A}}$, and satisfy $\lim _{\alpha} \int_{A} f_{\alpha} d \mu=\nu(A)$ for all $A \in \overline{\mathfrak{A}}$. Thus one gets a subnet $f_{\beta}$ of $f_{\alpha}$ such that $\lim _{\beta} \int_{A} f_{\beta} d \mu$ exists for all $A \in \mathfrak{A}$, i.e. $\lambda(A)=\lim _{\beta} \int_{A} f_{\beta} d \mu, A \in \mathfrak{A}$, defines some $\lambda \in b a_{+}(\Omega, \mathfrak{A})$ satisfying $\lambda \leqslant \mu$ and $\lambda|\overline{\mathfrak{A}}=\nu| \overline{\mathfrak{A}}$. Therefore, $\lambda=\nu$ is valid. The method of proof also yields the following characterization of the property of $\nu \in b a_{+}(\Omega, \mathfrak{A})$ to be uniquely determined among all $\lambda \in b a_{+}(\Omega, \mathfrak{A})$ satisfying $\lambda \lesssim \mu$ and $\lambda|\overline{\mathfrak{A}}=\nu| \overline{\mathfrak{A}}$ : For any net $f_{\alpha}$ of nonnegative functions that are primitive relative to $\mathfrak{A}$ and satisfy $\lim _{\alpha} \int_{A} f_{\alpha} d \mu=\nu(A)$ for all $A \in \overline{\mathfrak{A}}$ the $\operatorname{limit}_{\alpha} \lim _{\alpha} \int_{A} f_{\alpha} d \mu$ exists for any $A \in \mathfrak{A}$ and is equal to $\nu(A), A \in \mathfrak{A}$.

The second example concerns the special subalgebra $\overline{\mathfrak{A}}$ of invariant sets of $\mathfrak{A}$ in connection with the problem of finding conditions, which imply that the $f_{\alpha}$ of the underlying net of nonnegative functions of the theorem can be chosen to be already primitive relative to $\overline{\mathfrak{A}}$.

Example 2. Let $\Gamma$ denote a finite group of transformations $\gamma: \Omega \rightarrow \Omega$ satisfying $\gamma^{-1}(A) \in \mathfrak{A}, A \in \mathfrak{A}$, such that $\mu, \nu \in b a_{+}(\Omega, \mathfrak{A})$ are $\Gamma$-invariant, where $\lambda \in b a_{+}(\Omega, \mathfrak{A})$ is said to be $\Gamma$-invariant if and only if $\lambda\left(\gamma^{-1}(A)\right)=\lambda(A)$, $A \in \mathfrak{A}$, holds true. Under this general assumption there exists a net $f_{\alpha}$ of nonnegative functions that are primitive relative to $\overline{\mathfrak{A}}$, where $\overline{\mathfrak{A}}$ denotes the subalgebra $\{A \in \mathfrak{A}: \gamma(A)=A, \gamma \in \Gamma\}$ of $\Gamma$-invariant sets belonging to $\mathfrak{A}$, and satisfy $\lim _{\alpha} \int_{A} f_{\alpha} d \mu=\nu(A), A \in \mathfrak{A}$ if and only if $\nu \lesssim \mu$ is valid. This might be seen by starting from the inequality $\int f g d \mu \leq c<c+\varepsilon \leq \int g d \nu$ for all $f \in \mathfrak{F}$ of the first part of the proof of the theorem, where $\mathfrak{F}$ now stands for the set of all nonnegative functions that are primitive relative to $\overline{\mathfrak{A}}$. The general assumption implies that $g \in B(\Omega, \mathfrak{A})$ might be replaced by the function $h \in B(\Omega, \overline{\mathfrak{A}})$, which is defined by $h=\sum_{\gamma \in \Gamma} h \circ \gamma /|\Gamma| \quad(|\Gamma|$ cardinality of $\Gamma$ ). The remaining part of the proof is similar to the last part of the proof for the theorem. Example 2 admits as an application a characterization of the property of a $\Gamma$-invariant $\mu \in b a_{+}(\Omega, \mathfrak{A}), \mu(\Omega)=1$, that $\Gamma$ 's action is ergodic 
relative to $\mu$, i.e. $\mu \mid \overline{\mathfrak{A}}$ is $\{0,1\}$-valued. This property of $\mu$ is equivalent to the condition that there does not exist some $\Gamma$-invariant $\nu \in b a_{+}(\Omega, \mathfrak{A})$ satisfying $\nu \neq \mu, \nu(\Omega)=1$, and $\nu \lesssim \mu$. Clearly, if $\mu \mid \overline{\mathfrak{A}}$ is not $\{0,1\}$-valued, then $\nu$ defined by $\nu(A)=\mu\left(A \cap A_{0}\right) / \mu\left(A_{0}\right), A \in \mathfrak{A}$, where for $A_{0} \in \overline{\mathfrak{A}}$ the inequality $0<\mu\left(A_{0}\right)<1$ is valid, is $\Gamma$-invariant and satisfies $\nu \neq \mu, \nu \lesssim \mu$. Conversely, $\lim _{\alpha} \int_{A} f_{\alpha} d \mu=\nu(A), A \in \mathfrak{A}$, where $f_{\alpha}$ is a net of nonnegative functions that are primitive relative to $\overline{\mathfrak{A}}$, implies $\mu\left(\left\{f_{\alpha}=c_{\alpha}\right\}\right)=1$ for some $c_{\alpha} \geq 0$, from which $\mu(A)=\nu(A), A \in \mathfrak{A}$, follows.

\section{REFERENCES}

1. K. P. S. Bhaskara Rao and M. Bhaskara Rao, Theory of charges, Academic Press, New York, 1983.

2. N. Dunford and J. Schwartz, Linear operators, Part I, Interscience, New York, 1964.

WestFälische, Wilhelms-Universität, Institut für Mathematik, Einsteinstr. 62 D4400 MÜnster Germany 